\title{
Supplementary Material for: Detecting dynamical anomalies in time series from different palaeoclimate proxy archives using windowed recurrence network analysis
}

Jaqueline Lekscha ${ }^{1,2}$ and Reik V. Donner ${ }^{1,3}$

${ }^{1}$ Potsdam Institute for Climate Impact Research (PIK) - Member of the Leibniz Association, 14473 Potsdam, Germany

${ }^{2}$ Department of Physics, Humboldt University, 12489 Berlin, Germany

${ }^{3}$ Department of Water, Environment, Construction and Safety, Magdeburg-Stendal University of Applied Sciences, 39114 Magdeburg, Germany

Correspondence: lekscha@pik-potsdam.de

Additional figures
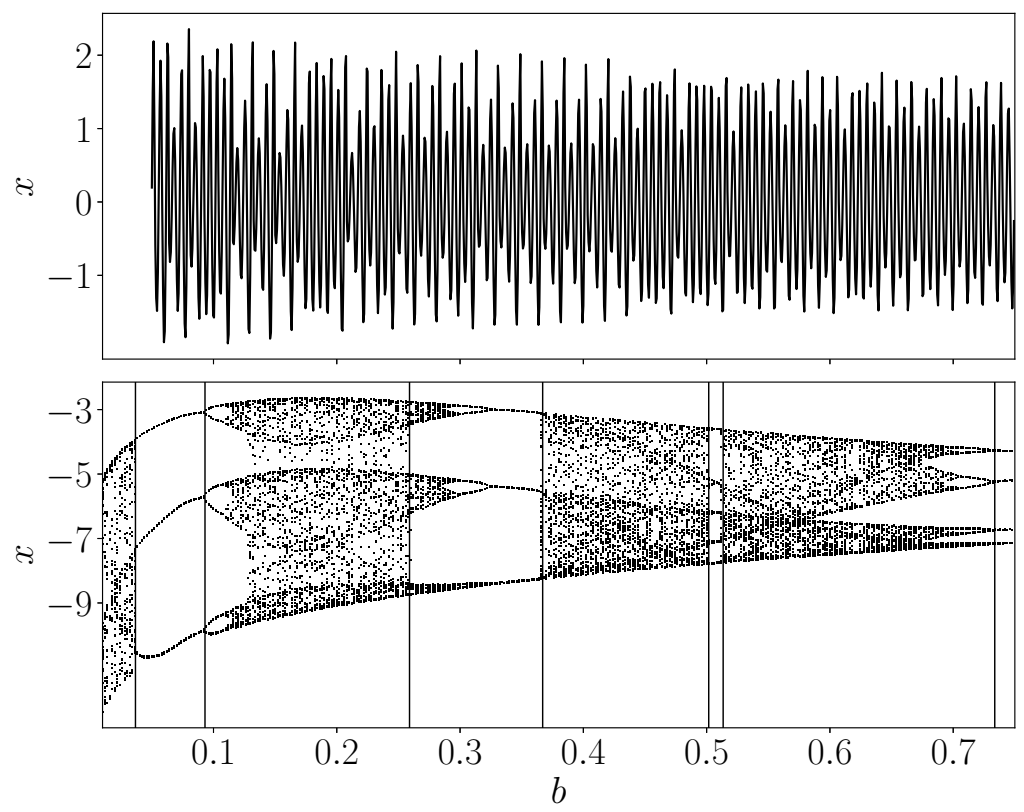

Figure S1. Transient time series of the $x$-component of the Rössler system with parameter $b$ varying in every integration step (top) and Feigenbaum diagram for corresponding stationary system (bottom). 
Gaussian white noise
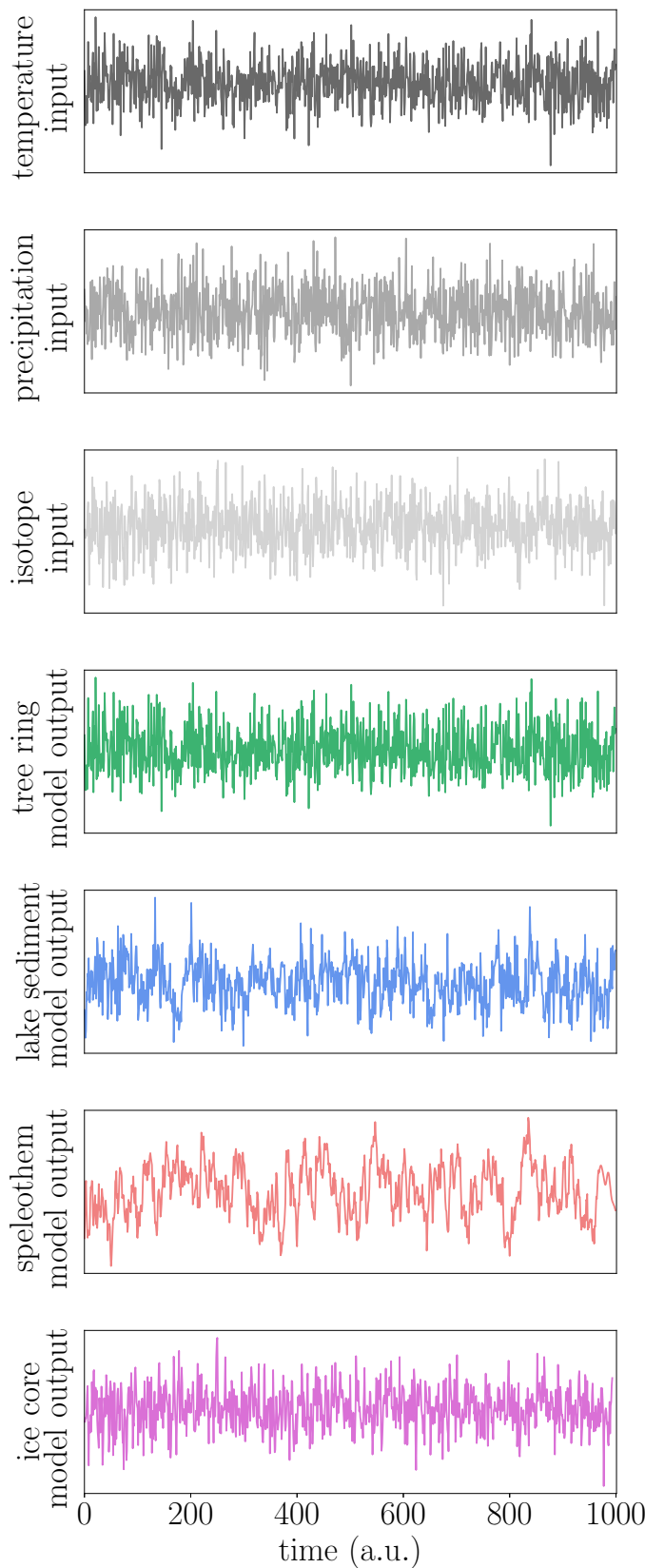

AR(1) process
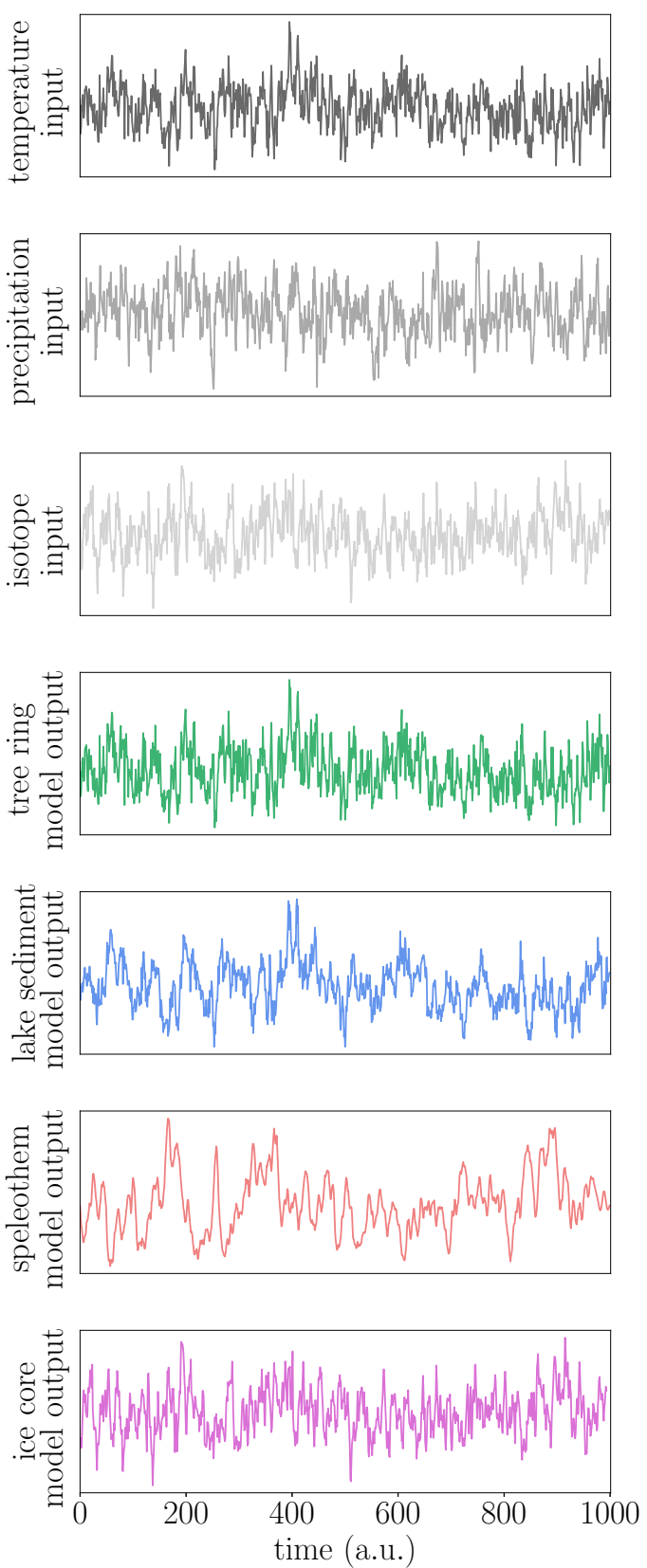

Figure S2. Annually resolved input time series for temperature, precipitation and isotopic composition and corresponding model output time series (top to bottom) for the two noise processes (left: Gaussian white noise, right: AR(1) process). 
non-stationary Rössler system
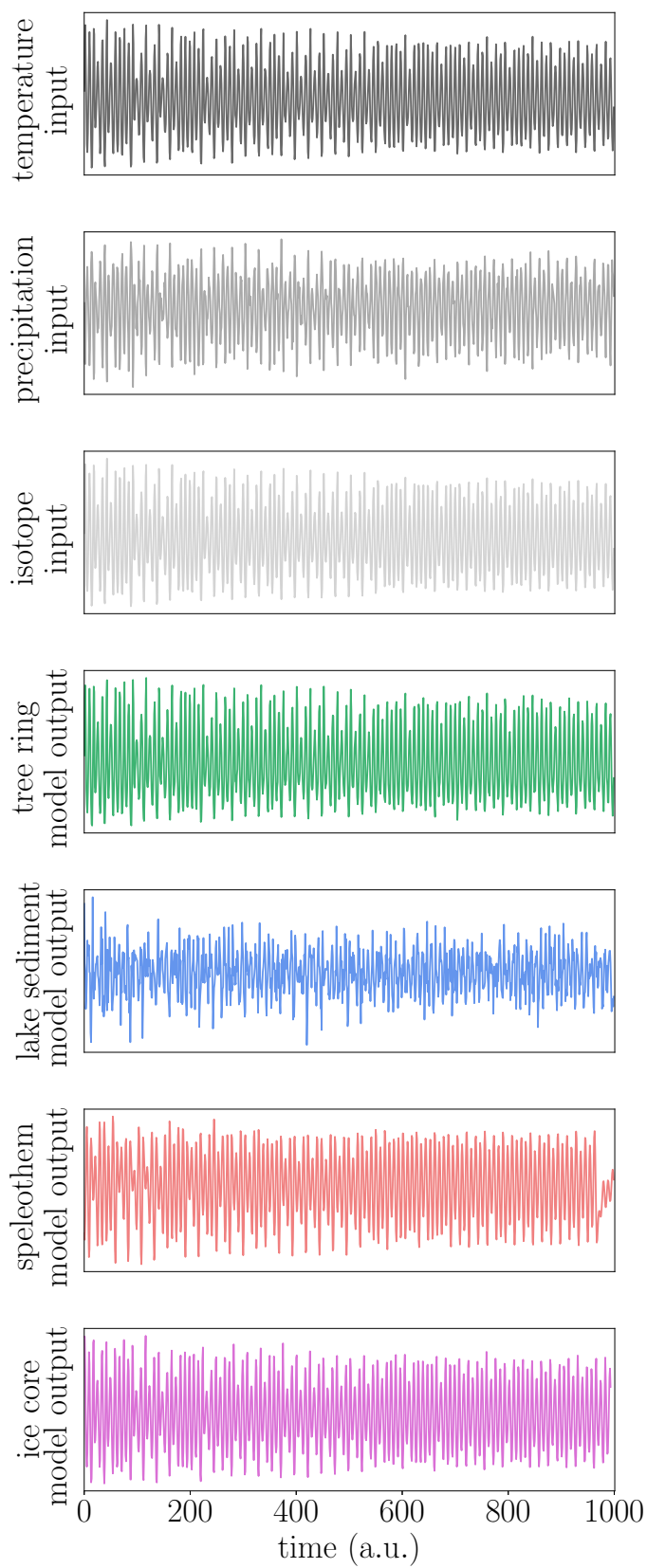

non-stationary Lorenz system
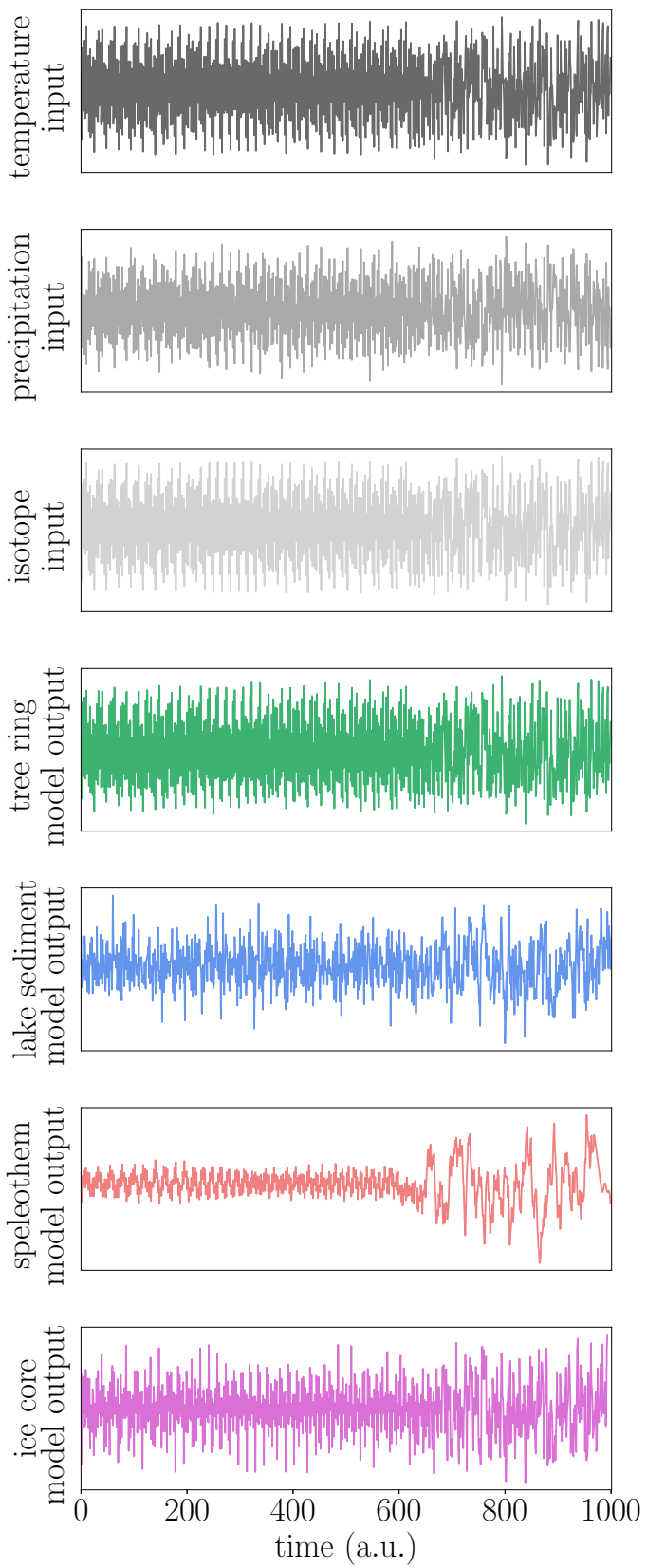

Figure S3. Same as in Fig. S2, but for the two deterministic non-stationary systems (left: non-stationary Rössler system, right: non-stationary Lorenz system). 

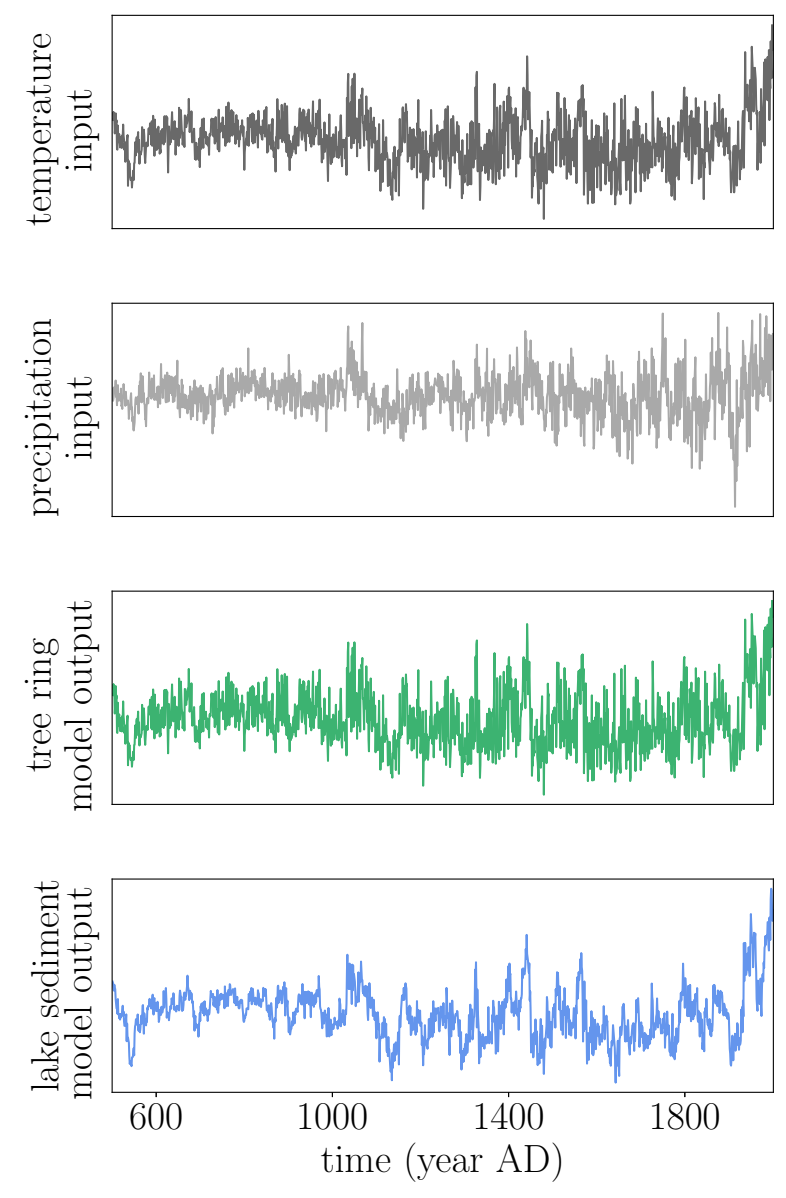

Figure S4. Annually resolved input time series for temperature and precipitation and corresponding model output time series (top to bottom) for the last millennium reanalysis data (Hakim et al., 2016; Tardif et al., 2019).

\section{Skewness of time series}

Table S1 gives the skewness of the distributions of the different time series where those values showing a significant deviation from the skewness of the corresponding input are marked in bold. To test whether the skewness values of the model output time

5 series differ significantly from that of the respective input, we create $N_{\mathrm{sk}}=10,000$ surrogate data sets for each of the input time series. For GWN and the AR(1) process, this is done by creating different realisations of the corresponding process according to the descriptions in Sec. 4, while for the other time series, the surrogates are created by adding white noise with signal-tonoise ratios of 25 for the model systems and 100 for the last millennium reanalysis data. Then, for each surrogate realisation, we calculate the skewness of the distribution and take the 0.5th and 99.5th percentile as confidence bounds resulting in the $99 \%$ 
Table S1. Skewness of the different input and proxy system model output time series with significant deviations of the model output time series from the corresponding input marked in bold.

\begin{tabular}{lrrrrr}
\hline input/output & GWN & AR(1) & ROS & LOR & LMR \\
\hline temperature & 0.00 & 0.07 & 0.21 & -0.02 & 0.33 \\
precipitation & 0.12 & -0.03 & -0.20 & 0.01 & 0.01 \\
isotopes & -0.06 & 0.12 & 0.22 & -0.02 & - \\
trw & $\mathbf{0 . 3 4}$ & $\mathbf{0 . 3 5}$ & $\mathbf{0 . 4 0}$ & $\mathbf{0 . 2 6}$ & $\mathbf{0 . 6 3}$ \\
lak & 0.11 & 0.23 & $-\mathbf{0 . 0 5}$ & $\mathbf{0 . 0 1}$ & $\mathbf{0 . 7 1}$ \\
spt & -0.04 & 0.29 & $-\mathbf{0 . 2 7}$ & $\mathbf{0 . 2 6}$ & - \\
ice & -0.01 & 0.15 & $\mathbf{0 . 1 5}$ & $-\mathbf{0 . 0 5}$ & - \\
\hline
\end{tabular}

10 confidence intervals of $[-0.20,0.20]$ for GWN, $[-0.29,0.29]$ for the AR(1) process, $[0.20,0.23]$ for the non-stationary Rössler system, $[-0.03,0.00]$ for the non-stationary Lorenz system, $[0.32,0.34]$ for the last millennium reanalysis temperature, and $[0.00,0.02]$ for the last millennium reanalysis precipitation. As precipitation is proportional to the negative temperature and isotopes, the corresponding confidence interval for the Rössler system is $[-0.23,-0.20]$ and for the Lorenz system $[0.00,0.03]$. 


\section{References}

Hakim, G. J., Emile-Geay, J., Steig, E. J., Noone, D., Anderson, D. M., Tardif, R., Steiger, N., and Perkins, W. A.: The last millennium climate reanalysis project: Framework and first results, Journal of Geophysical Research: Atmospheres, 121, 6745-6764, https://doi.org/10.1002/2016JD024751, 2016.

Tardif, R., Hakim, G. J., Perkins, W. A., Horlick, K. A., Erb, M. P., Emile-Geay, J., Anderson, D. M., Steig, E. J., and Noone, D.: Last Millennium Reanalysis with an expanded proxy database and seasonal proxy modeling, Climate of the Past, 15, 1251-1273, https://doi.org/10.5194/cp-15-1251-2019, 2019. 